\title{
Henri DORVIL
}

Travailleur social, professeur, École de Travail social, UQÀM

(1981)

\section{"La psychiatrie au Québec: réalité d'hier, pratique d'aujourd'hui."}

Un document produit en version numérique par Jean-Marie Tremblay, bénévole, Professeur sociologie au Cégep de Chicoutimi

Courriel: jean-marie_tremblay@uqac.ca

Dans le cadre de "Les classiques des sciences sociales"

Site web: http://classiques.uqac.ca/

Une bibliothèque fondée et dirigée par Jean-Marie Tremblay, sociologue

Une collection développée en collaboration avec la Bibliothèque

Paul-Émile-Boulet de l'Université du Québec à Chicoutimi

Site web: http://bibliotheque.uqac.ca/ 


\section{Politique d'utilisation de la bibliothèque des Classiques}

Toute reproduction et rediffusion de nos fichiers est interdite, même avec la mention de leur provenance, sans l'autorisation formelle, écrite, du fondateur des Classiques des sciences sociales, Jean-Marie Tremblay, sociologue.

Les fichiers des Classiques des sciences sociales ne peuvent sans autorisation formelle:

- être hébergés (en fichier ou page web, en totalité ou en partie) sur un serveur autre que celui des Classiques.

- servir de base de travail à un autre fichier modifié ensuite par tout autre moyen (couleur, police, mise en page, extraits, support, etc...),

Les fichiers (.html, .doc, .pdf., .rtf, .jpg, .gif) disponibles sur le site Les Classiques des sciences sociales sont la propriété des Classiques des sciences sociales, un organisme à but non lucratif composé exclusivement de bénévoles.

Ils sont disponibles pour une utilisation intellectuelle et personnelle et, en aucun cas, commerciale. Toute utilisation à des fins commerciales des fichiers sur ce site est strictement interdite et toute rediffusion est également strictement interdite.

L'accès à notre travail est libre et gratuit à tous les utilisateurs. C'est notre mission.

Jean-Marie Tremblay, sociologue

Fondateur et Président-directeur général, LES CLASSIQUES DES SCIENCES SOCIALES. 
Cette édition électronique a été réalisée Jean-Marie Tremblay, bénévole, professeur de soins infirmiers retraitée de l'enseignement au Cégep de Chicoutimi

Courriel: jean-marie_tremblay@uqac.ca

à partir du texte de :

Henri Dorvil, “La psychiatrie au Québec : réalité d'hier, pratique d'aujourd'hui”. Un article publié dans L'intervention sociale. Actes du Colloque annuel de l'ACSALF, colloque 1981. Textes publiés sous la direction de Micheline Meyer-Renaud et Alberte Le Doyen, pp. 111-131. Montréal : Les Éditions coopératives Albert Saint-Martin, 1982, 384 pp.

M. Henri Dorvil, travailleur social, enseigne à l’École de travail sociale de l’UQÀM.

[Autorisation formelle de l’auteur accordée le 18 mai 2008.]

Courriel : dorvil.henri@uqam.ca

Polices de caractères utilisée :

Pour le texte: Times New Roman, 14 points.

Pour les citations : Times New Roman 12 points.

Pour les notes de bas de page : Times New Roman, 12 points.

Édition électronique réalisée avec le traitement de textes Microsoft Word 2004 pour Macintosh.

Mise en page sur papier format : LETTRE (US letter), 8.5’’ x 11'’)

Édition numérique réalisée le 17 mai 2008 à Chicoutimi, Ville de Saguenay, province de Québec, Canada. 


\section{Henri Dorvil}

Travailleur social, professeur, École de Travail social, UQÀM

\section{"La psychiatrie au Québec : réalité d'hier, pratique d'aujourd'hui”}

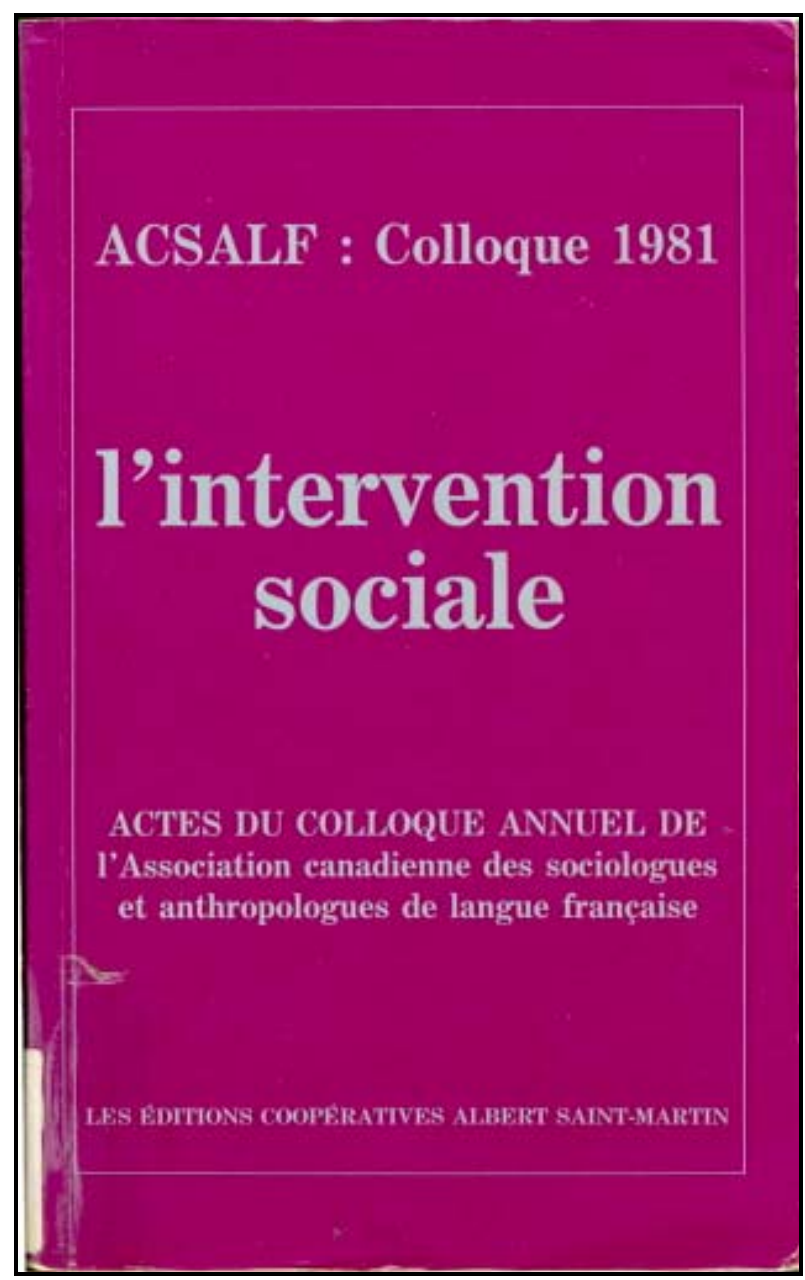

Un article publié dans L'intervention sociale. Actes du Colloque annuel de l'ACSALF, colloque 1981. Textes publiés sous la direction de Micheline MeyerRenaud et Alberte Le Doyen, pp. 111-131. Montréal : Les Éditions coopératives Albert Saint-Martin, 1982, 384 pp. 


\section{Table des matières}

$\underline{\text { Introduction }}$

La période politico-religieuse ou pé-asilaire (avant 1845)

La période asilaire ou la période du professionnalisme d'affaires

(1845-1895)

La période neuro-hospitalière ou franco-religieuse (1895-1962)

La période communautaire

$\underline{\text { Réflexions sur les pratiques actuelles }}$

Bibliographie

Tableau 1. Les principales caractéristiques de l'évolution du système psychiatrique au Québec 


\section{Henri Dorvil,}

Travailleur social, professeur, École de Travail social, UQÀM

“La psychiatrie au Québec : réalité d'hier, pratique d'aujourd'hui”.

Un article publié dans L'intervention sociale. Actes du Colloque annuel de l'ACSALF, colloque 1981. Textes publiés sous la direction de Micheline MeyerRenaud et Alberte Le Doyen, pp. 111-131. Montréal : Les Éditions coopératives Albert Saint-Martin, 1982, 384 pp.

\section{Introduction}

De nombreux auteurs (M. Foucault (1966), T. Szasz (1976), R. Castel (1979), etc.) nous ont appris que l'idée de définir la folie comme une maladie mentale est à vrai dire une idée relativement récente, c'est-à-dire qui date du 17e siècle environ. Et même, il faudra attendre le milieu du 19e siècle pour que cette idée d'assimiler la folie à une maladie débouche sur la psychiatrie comme science et comme pratique sociale.

Auparavant, du moins dans le monde occidental, la folie est pour l'essentiel éprouvée à l'état libre ; le fou circule partout où il veut, il va, il vient ; il fait partie du décor pour ainsi dire et il est intégré à la vie quotidienne ainsi qu'à la culture commune (on retrouve le personnage du fou dans la peinture, le théâtre, l'architecture par exemple). Avec le développement du capitalisme (vers le milieu du 17e siècle en Europe) on commence à enfermer les «fous » avec l'ensemble des « marginaux » de l'époque, prêtres défroqués, libertins, pauvres, handicapés, vieillards... La " tare » commune de ces gens est précisément de ne pas être productifs, de ne pas travailler. C'est d'ailleurs ce qui 
explique qu'à l'intérieur de ce qu'on appelle l'hôpital général, ceux qui sont enfermés sont soumis au travail forcé. C'est là le tribut à payer à la morale bourgeoise qui s'implante : «l'oisiveté devient la mère de tous les vices ».

Donc, on peut avancer que de façon générale, les sociétés occidentales ont été largement caractérisées par une longue tradition de renfermement punitif d'une catégorie sociale appelée «malades mentaux ». Toutefois, au fil de l'évolution, chaque société a trouvé une manière originale de rompre à un moment donné avec le système asilaire.

En France par exemple ce mouvement de rupture s'amorce aux lendemains de la seconde guerre mondiale. Durant l'occupation allemande, beaucoup de malades mentaux sont morts d'inanition dans les asiles ; les psychiatres français ont assisté impuissants à ce spectacle et dès la paix revenue ont décidé irréversiblement de sortir la psychiatrie de ce carcan, ou bien en transformant l'hôpital psychiatrique en organisation moderne de soins axée sur des techniques relationnelles, et c'est la psychiatrie institutionnelle, ou bien en développant une psychiatrie hors des murs de l'hôpital axée sur toute une gamme de soins destinés à la population, et c'est la psychiatrie de secteur.

Aux U.S.A., la santé mentale communautaire s'est développée à l'ombre du mouvement général de santé publique qui avait réussi à endiguer des fléaux publics comme la fièvre jaune, la tuberculose, etc. D'où les « mental health clinics ». Plus tard, vers les années quarante, comme la psychiatrie américaine avait réussi à juguler la névrose de combat, problème très coûteux pour l'économie militaire, les psychiatres ont vite conclu que toutes les maladies psychiatriques ne sont que « réactions » et sont susceptibles d'être guéries. Cette idée et l'arrivée des drogues psychotiques ont suffi pour transformer le visage des asiles et pour faire sortir les malades de ces institutions. De plus, la Commission conjointe sur la maladie mentale et la santé mentale et le discours du président Kennedy au Congrès américain plaident en fa- 
veur du malade mental inconsciemment rejeté par sa famille, par ses voisins et par les professionnels.

Le Québec va connaître sensiblement la même évolution, avec bien sûr un certain retard par rapport au contexte européen et aussi avec certaines particularités liées au contexte socio-culturel d'ici (voir plus loin). L'analyse de cette évolution est intéressante à plus d'un titre car, comme l'a signalé $\mathrm{H}$. Wallot (1979), l'histoire de la folie dans une société donnée présente souvent un double intérêt. D'abord elle révèle « la nature des répressions » que cette société se donne et nous informe par le fait même "sur l'évolution des groupes au pouvoir dans cette société ». Cette histoire permet ainsi de constater que « les buts des organisations qui prennent en charge la folie, se concrétisent différemment selon la définition sociale de la folie à une époque et selon les intérêts en place » (p. 102). C'est dans cette perspective que l'auteur a retracé l'histoire de la psychiatrie au Québec, en dégageant cinq grandes périodes: a) la période politico-religieuse ou pré-asilaire (avant 1845) ; b) la période asilaire ou celle du professionnalisme d'affaires (1845-1895); c) la période neuro-hospitalière ou francoreligieuse (1895-1962) ; la période psychiatrique (1962-1971) et finalement la période sociale (1971-1978). L'auteur conclut sur la période actuelle, qu'il identifie comme étant une période de «récupération communautaire ».

Dans la même perspective, on doit signaler l'excellente étude de Françoise Boudreau (1978) sur l'évolution du système psychiatrique au Québec, où sont dégagées trois grandes périodes : 1) du début de la colonisation à la révolution tranquille (1960), 2) de 1960 à 1970 et 3) de 1970 à 1978 (date de parution de l'article). Pour chacune de ces grandes étapes, l'auteur tente de dégager a) la principale caractéristique du système en place; b) les principaux promoteurs de ce système ; c) l'idéologie dominante ; d) le but officiel déclaré ; e) la représentation de la situation et finalement $\mathrm{f}$ ) le modèle d'intervention privilégié (voir le tableau ci-dessous ; traduction libre). De même, la récente analyse des psychiatres G. Aird et A. Amyot (1980) sur l'évolution 
de la psychiatrie communautaire au Québec reprend sensiblement cette division en trois grandes étapes puisqu'ils évoquent : 1) la période de la psychiatrie asilaire, de 1845 à 1960, 2) la révolution psychiatrique au Québec, de 1960 à 1970, et finalement 3) la période de crise, de 1971 à 1977.

Comme on peut le constater, ces tentatives de «typologie " se recoupent sensiblement ; c'est pourquoi, pour nos besoins, nous allons tenter de les résumer brièvement, tout en étant bien conscients qu'il s'agit là de grandes étapes et que la réalité historique n'est sans doute pas aussi linéaire qu'on le laisse entendre.

\section{La période politico-religieuse ou pé-asilaire (avant 1845)}

$\underline{\text { Retour à la table des matières }}$

Comme point de départ il faut citer les excellents travaux de A. Paradis et de son équipe $(1976,1978)$ et de H. Wallot (1979) sur le développement des " asiles » psychiatriques au Québec. Ainsi, après avoir évoqué les effets de la Conquête, le second souligne qu'avant 1845, au Québec, «la folie bien qu'émergeant comme entité distincte faisait partie d'un continuum de la déviance sociale allant de la criminalité jusqu'à l'impiété, l'hérésie ou la possession » (p. 103). Les institutions d'hébergement et d'internement (les prisons, les hôpitaux, etc.) de l'époque assumaient un rôle de répression et de contrôle social sous l'égide de l'Église ; ainsi, à l'Hôtel-Dieu de Québec, « on fit des exorcismes et on fonda une maison de force " pour les femmes "furieuses » ou de «mauvaise vie » (p. 103). Vers 1801, le gouvernement commence à assumer les frais d'entretien des malades mentaux du Bas-Canada. 
Tableau 1

Les principales caractéristiques de l'évolution du système psychiatrique au Québec

$\underline{\text { Retour à la table des matières }}$

\begin{tabular}{|c|c|c|c|}
\hline \multirow{2}{*}{ Les variables } & \multicolumn{3}{|c|}{ Les périodes } \\
\hline & Avant 1960 & $1960-1970$ & $1970-1978$ \\
\hline Le système & $\begin{array}{l}\text { Système des « asi- } \\
\text { les » }\end{array}$ & $\begin{array}{l}\text { Système « psychia- } \\
\text { trique » }\end{array}$ & $\begin{array}{l}\text { Système des affaires } \\
\text { sociales (« health and } \\
\text { welfare » system) }\end{array}$ \\
\hline \multirow[t]{2}{*}{ Les promoteurs } & $\begin{array}{l}\text { Les « traditionnalis- } \\
\text { tes » }\end{array}$ & Les «modernistes» & \\
\hline & $\begin{array}{l}\text { • les communautés } \\
\text { religieuses } \\
\text { • les médecins « tra- } \\
\text { ditionnels » }\end{array}$ & $\begin{array}{l}\text { • les jeunes psychia- } \\
\text { tres }\end{array}$ & $\begin{array}{l}\text { - Les technocrates } \\
\text { - Les administrateurs } \\
\text { - Les « planificateurs } \\
\text { sociaux » }\end{array}$ \\
\hline $\begin{array}{l}\text { L'idéologie domi- } \\
\text { nante }\end{array}$ & $\begin{array}{l}\text { La folie n'est pas gué- } \\
\text { rissable }\end{array}$ & $\begin{array}{l}\text { La maladie mentale } \\
\text { est une maladie } \\
\text { comme une autre }\end{array}$ & $\begin{array}{l}\text { La santé mentale est un } \\
\text { droit pour tous }\end{array}$ \\
\hline Le but officiel & $\begin{array}{l}\text { Travail terrestre pour } \\
\text { une meilleure place } \\
\text { au ciel }\end{array}$ & $\begin{array}{l}\text { Traitement et guéri- } \\
\text { son }\end{array}$ & $\begin{array}{l}\text { Complémentarité entre } \\
\text { santé physique et santé } \\
\text { mentale }\end{array}$ \\
\hline $\begin{array}{l}\text { Représentation de } \\
\text { la situation }\end{array}$ & $\begin{array}{l}\text { Les communautés } \\
\text { religieuses sont les } \\
\text { plus compétentes } \\
\text { pour soigner les ma- } \\
\text { lades }\end{array}$ & $\begin{array}{l}\text { Confiance dans les } \\
\text { experts }\end{array}$ & $\begin{array}{l}\text { Tout le monde peut et } \\
\text { doit contribuer à la gué- } \\
\text { rison. Le gouvernement } \\
\text { intervient au nom de la } \\
\text { collectivité }\end{array}$ \\
\hline $\begin{array}{l}\text { Le modèle d'inter- } \\
\text { vention privilégié }\end{array}$ & $\begin{array}{l}\text { • Modèle de gardien- } \\
\text { nage (« custodial } \\
\text { model ») } \\
\text { • Traitement résiden- } \\
\text { tiel }\end{array}$ & $\begin{array}{l}\text { • Modèle curatif } \\
\text { • Traitement psy- } \\
\text { chiatrique individua- } \\
\text { lisé }\end{array}$ & $\begin{array}{l}\text { • Modèle préventif } \\
\text { - Traitement intégré de } \\
\text { santé publique }\end{array}$ \\
\hline
\end{tabular}


Toutefois, comme cela s'était passé en Europe, on va de plus en plus s'opposer à la mixité des fous et des prisonniers et ce, « au nom d'une nouvelle compréhension de l'aliénation » qui sert d'idéologie à la nouvelle élite professionnelle montante représentée notamment par les médecins. C'est le passage d'une phase d'internement indifférencié à une phase d'internement spécialisé. Ainsi, l'Ontario en vient à créer en 1841 le premier asile étatique au Canada, ce qui a pour effet de faire entrer, aux côtés des autorités civiles, les professionnels de la médecine dans le champ du contrôle social. Dans ce sens, ils vont rapidement établir la distinction classique entre les malades curables et les malades incurables. Malgré les nombreuses techniques dites scientifiques (les saignées, etc.) et les échecs répétés, les médecins vont justifier alors « leurs interventions surtout au niveau hygiénique et moral » (p. 104). Comme le nombre de malades « incurables » (sic) augmente sans cesse, on va se mettre à rêver d'un asile permanent qui, par sa « modernité », est censé, comme l'a bien montré A. Paradis (1978), régler tous les problèmes.

C'est donc une période où la primauté de la charité chrétienne et de la philanthropie privée est clairement affirmée. L'action sociale vis-àvis des «fous » est assez typique de la philosophie sociale de l'époque. On assiste à un « dosage » de pratiques d'assistance et de répression ; l'idéologie morale est également très présente dans le nouveau champ d'intervention qui s'élabore à cette époque, celui de l'internement des " aliénés ». Le Québec n'a donc pas échappé à cette solution de «bonne conscience » qui a consisté à isoler les malades mentaux du reste de la société par leur internement dans des asiles.

Rappelons brièvement à ce propos que dès le début du 18' siècle Mgr de St-Vallier fait construire des « loges » pour aliénés violents sur les terrains de l'Hôpital Général de Québec. Jusqu'à la fin du 18' siècle, l'entretien des aliénés relèvera de la charité privée, encouragée à l'occasion par une aide financière de la Cour du Gouverneur. En 1801, une première loi est votée (« Acte pour le soulagement des dé- 
rangés dans leur esprit »), qui vise simplement à aider financièrement les hôpitaux généraux de Montréal, Québec et Trois-Rivières.

Après l'Acte d'Union (en 1841), c'est le triomphe de l'humanisme anglais, avec ses critiques virulentes des conditions antérieures. Il y a aussi une certaine volonté de législation en 1845 ; cependant, il n'est pas question d'un asile d'État, comme à Toronto (1841), mais plutôt de la mise sur pied d'un système d'affermage, c'est-à-dire d'un contrat avec l'entreprise privée (Dr Douglas). À la même époque c'est la création au Québec de l'asile de Beauport (1845), qui va vite devenir une institution encombrée et va fonctionner selon la règle du profit maximum.

A. Paradis (1976) a bien fait saisir la relation entre l'avènement de l'institution asilaire et les processus de l'industrialisation en cours au Québec. D'où la fonction "politique » de l'asile vis-à-vis du sousprolétariat naissant. De plus, il analyse l'asile naissant comme une institution politique : en montrant la structure interne (90\% de la clientèle est composée de Canadiens français et d'Irlandais) en regard des rapports sociaux globaux, il conclut à l'impossibilité de réaliser le rapport thérapeutique entre médecin et patient. Il fait aussi l'hypothèse d'une relation entre le passage du capitalisme commercial au capitalisme industriel et le discours sur la maladie mentale. La « nouvelle » philosophie de traitement fait appel aux notions de travail comme thérapie, d'organisme, de bonheur et d'abolition de la répression. Dès cette époque les Appareils Idéologiques d'État prennent le pas sur les appareils répressifs et la violence physique se transforme en violence morale. L'efficacité du pouvoir réside désormais dans son invisibilité et nous assistons à l'élaboration, via la thérapie, d'un processus d'intériorisation des normes dominantes chez les malades (les dominés).

De plus, A. Paradis (1976) se demande pourquoi l'asile de Beauport était privé. Parce que, répond-il, la catégorie de fous susceptibles d'internement à Beauport n'intéresse aucune des deux communautés. Au Canada français le fou a une certaine place dans la communauté 
traditionnelle, alors que du côté du Canada anglais on se montre peu intéressé. La petite bourgeoisie professionnelle va jouer un certain rôle dans la promotion de l'asile, principalement parce qu'elle est « dérangée » dans son travail quotidien. De plus, le fossé dans la relation médecin-malade conduit finalement à une stagnation du discours et de la thérapie. Le " traitement » est en effet marqué par une conception «médicalisée » du problème, d'où la négation de sa dimension socioculturelle et politique. Il s'agit d'un discours « emprunté » et caractérisé essentiellement par un traitement physique à incidence morale qui vise la « libération » de l'aliéné en proposant des modes "d'occupation » et une saine alimentation. Il s'agit aussi de recourir à une pratique de psychothérapie élémentaire dans les rapports gardiensmalades : douceur, bienveillance, paternalisme, etc., ainsi qu'au conditionnement quotidien par les travaux champêtres, les pratiques religieuses et les amusements. C'est ce qu'on appelle l'humanisation du traitement. Finalement, le système binaire de classement entre curables et incurables tient lieu de nosographie... Mais le gonflement rapide de la clientèle asilaire amènera une saturation qui suscitera une demande pour une institution plus spécialisée «pour les idiots et les aliénés sans espoir ». Et l'utopie de l'institution miracle reprend à nouveau.

\section{La période asilaire ou la période du professionnalisme d'affaires (1845-1895)}

Retour à la table des matières

Ainsi donc, après de multiples péripéties (pétitions, enquêtes, etc.) le premier asile est fondé à Beauport en 1845. Il deviendra au début du 20' siècle l'Hôpital St-Michel-Archange. Par la suite, d'autres institutions asilaires sont fondées au 19' siècle, dont les plus importantes sont situées à Montréal : St-Jean-De-Dieu (1873) et Douglas (1889). Il 
faut rappeler que la question des asiles va alors susciter des débats politiques et idéologiques importants : il s'agissait de savoir si les communautés religieuses devaient prendre le contrôle des institutions asilaires francophones et quelle serait la place de l'État et de la médecine dans ce système. Vers 1890 commence un long monopole des communautés religieuses qui va persister jusqu'au début des années 1960. Mais nous anticipons sur la suite.

Revenons un peu en arrière pour souligner qu'une fois l'Union du Bas-Canada et du Haut-Canada acquise (1841), on a jugé qu'il ne serait plus question de dépenser pour des aliénés du Québec et encore moins de construire un asile permanent comme en Ontario. De plus, au lieu de s'allier à l'Église, le nouveau pouvoir britannique va plutôt s'adresser à un professionnel anglais, le Dr Douglas, homme d'affaires de surcroît, afin de transiger des contrats de service pour l'asile de Beauport. Selon H. Wallot (1979), la nomination du Dr Douglas marque « l'entrée en scène de la profession médicale, du capitalisme, du colonialisme dans l'histoire de la folie au Québec » (p. 104).

De leur côté, F. Harvey (1975) et R. Samuel (1974) ont eux aussi souligné que le rôle de l'asile à cette époque consistait essentiellement à interner le plus grand nombre d'aliénés afin de «protéger la société » et qu'en conséquence l'asile était considéré comme une «maison de refuge » plutôt que comme une institution de santé, car le traitement y était davantage de nature hygiénique et morale que proprement médicale. De plus, R. Samuel (1974) a souligné que les asiles fonctionnaient alors sur le "système d'affermage ", qui laissait une complète autonomie aux propriétaires. Ce système semblait économique pour la province et lucratif pour les propriétaires. Selon R. Samuel, les premières attaques contre ce "système d'affermage " se situent vers les années 1880. Certaines personnalités insinuent que le système d'affermage est synonyme de profit pour les propriétaires et que le traitement médical est réduit à sa plus simple expression (p. 17). Jusqu'en 1880, la législation sur les asiles était à peu près inexistante, ce qui traduit, selon R. Samuel, " une certaine indifférence du gouverne- 
ment » (p. 18). Les asiles sont surtout l'affaire des propriétaires et les inspecteurs du gouvernement ont peu d'influence. Les contrats à long terme tiennent lieu de politique. Étant donné l'augmentation des coûts et des subsides, le gouvernement crée la loi de 1885, qui vise la création d'un bureau médical dont la majorité des membres serait nommée par le gouvernement. Le gouvernement veut donc assumer le contrôle médical des asiles. L'introduction de la loi va " politiser » davantage la question des asiles, suscitant de nombreux débats tant parlementaires que publics (voir les journaux) ; la situation est telle que le premier ministre Mercier doit instituer une commission royale d'enquête en 1887.

Par ailleurs, Alain Fugère (1978) a noté à propos de la littérature publiée au Québec au 19' siècle l'absence de texte théorique ou analytique concernant la folie ; presque tout ce qui s'écrit concerne plutôt l'administration des institutions asilaires et particulièrement leur financement. Quant aux stratégies thérapeutiques, on y fait seulement allusion car elles étaient peu présentes dans les préoccupations des médecins de l'époque. Henry Howard (1815-1887) a cependant constitué une exception, puisqu'il a publié de nombreux articles et même un volume sur le sujet. L'intérêt du texte d'A. Fugère est de montrer que « les enjeux sociaux et politiques déterminent largement l'élaboration théorique » (p. 28). Même si Howard se réfère à la Science pour justifier son discours, il n'en élabore pas moins, comme le montre A. Fugère, un discours humaniste à forte saveur religieuse ; toutefois ce discours n'est pas sans lien avec les conflits socio-politiques de l'époque, particulièrement en ce qui touche le rôle de l'Église dans la société civile ainsi que les querelles autour des écoles séparées et gratuites. Bref, Howard tente de "médicaliser » le discours de la folie et d'assurer la reconnaissance professionnelle du métier d'aliéniste. Fugère a raison de conclure que le discours pré-psychiatrique québécois, même à son meilleur, n'échappe pas «à l'emprise des valeurs régnantes et des intérêts professionnels » (p. 43). 


\section{La période neuro-hospitalière ou franco-religieuse (1895-1962)}

$\underline{\text { Retour à la table des matières }}$

Après de multiples débats autour du contrôle des asiles, et suite à la Commission royale d'enquête de 1893, le gouvernement du Québec négocie la prise en charge de l'asile de Beauport par les Soeurs de la Charité, moyennant un per capita annuel de 100\$ (contre 140\$ au temps du Dr Douglas et 116\$ pour l'hôpital protestant de Verdun). En 1923, à Québec, on crée la clinique Roy-Rousseau, en l'honneur de la petite élite clérico-bourgeoise (Mgr Roy est alors recteur de l'Université Laval et le Dr Rousseau est doyen de la Faculté de médecine). Cette clinique est bâtie par les patients de l'hôpital, avec l'aide d'un architecte, et doit s'occuper des patients dont l'état n'exige pas soit un internement, soit un traitement de longue durée. Toutefois, comme le souligne $\mathrm{H}$. Wallot (1979), la clinique dut se financer elle-même et devint rapidement « un établissement de classe (...) qui desservit anonymement et sans dossier les personnalités religieuses et politiques » (p. 108).

Au niveau thérapeutique, le modèle médical de la maladie mentale était prédominant, de sorte qu'il s'agissait surtout d'un « modèle neurologique, modèle descriptif et fataliste où le traitement se limitait à un support médical » (p. 108) sans aborder vraiment la dimension psychiatrique. Bref, il s'agissait d'un modèle professionnel valorisant la dimension hébergement-internement aux dépens de la dimension traitement.

Par ailleurs, comme le souligne H. Wallot, « si la société a intérêt à protéger cet hôpital, si les médecins y trouvent la justification d'une participation au pouvoir de contrôle social au nom d'un savoir médi- 
cal, les seules personnes qui trouvent un intérêt personnalisé dans les malades sont les religieuses qui y voient des âmes à sauver » (p. 108). Dans cette perspective, il n'est pas trop surprenant de retrouver dans ce type d'institution la forte présence d'un traitement d'ordre moral où l'on prêche aux malades la chasteté, la pauvreté et l'obéissance. À vrai dire, les religieuses ne font que répéter ce qu'on leur a inculqué.

En somme, on peut dire que depuis la Confédération le développement de la psychiatrie au Québec a suivi deux directions assez distinctes et surtout très révélatrices du clivage religieux, culturel et économique et politique entre Canadiens anglais et Canadiens français. Du côté francophone, les asiles sont pris en charge par les communautés religieuses catholiques avec ce que cela implique de moralisme et de résignation, alors que du côté anglophone, les moyens financiers (privés) aidant, les asiles se tournent vers la nouvelle "science » qu'est la psychiatrie. Ainsi donc, on a à cette époque, d'un côté une société rurale et traditionnelle, de l'autre une société résolument orientée vers le capitalisme industriel avec des valeurs reliées à l'essor économique qui sont de l'ordre du «modernisme » et du " scientisme ». C'est un peu ce qui explique ce que l'on a par la suite appelé « l'avance » des institutions psychiatriques anglophones. Du côté francophone, il faudra attendre la révolution tranquille et donc les années soixante pour que la situation change dans les institutions pour " aliénés » et pour que l'État intervienne dans ces institutions et se préoccupe du sort de ces malades. Les Québécois francophones allaient passer de la campagne à la ville, de la société traditionnelle à la société « libérale ». L'ère des réformes arrivait et dans le domaine de la santé mentale, on allait passer rapidement, à coup de législations, de l'ère asilaire à l'ère psychiatrique, pour arriver aujourd'hui dans ce que certains ont appelé l'ère communautaire. 


\section{La période communautaire}

$\underline{\text { Retour à la table des matières }}$

Cette période débute par une phase de démocratisation. L'appareil de soins psychiatriques cesse d'être la chasse gardée des communautés religieuses, des médecins traditionalistes, voire des psychiatres québécois modernes qui reviennent de leur cycle de formation à l'étranger, aux États-Unis, en France ou dans les deux pays. C'est aussi l'affaire avant tout des patients eux-mêmes, les psychiatrisés, ainsi que de toute une gamme de professionnels de la santé, personnel infirmier (gardes-malades, préposés), travailleurs sociaux, psychologues, ergothérapeutes, conseillers en orientation professionnelle, en éducation physique, sociologues, etc. Le détonateur de la révolution psychiatrique est d'ailleurs venu principalement d'un livre rédigé par un ex-patient : Les Fous crient au secours, de Jean-Charles Pagé, publié en 1961 avec l'imprimatur du directeur du Département de psychiatrie de l'Université de Montréal, ancien médecin traitant de l'auteur. Ce cri d'alarme poussé à l'aube de la révolution tranquille s'inspire d'un mouvement de réveil, de turbulence et de contestation du monde à prédominance religieuse et rurale de l'époque. Dans la mesure où les fous constituent des otages dans une société donnée, tout soubresaut a des effets sur le sort des malades mentaux. Ainsi, beaucoup d'hôpitaux psychiatriques changent de nom et même de vocation pour mieux refléter les aspirations de l'heure. La commission d'étude des hôpitaux psychiatriques créée par le gouvernement du Québec en réponse aux propos de Jean-Charles Pagé et Camille Laurin recommande l'arrêt du dernier asile à Sherbrooke. Si la commission tolère la poursuite des travaux de construction des asiles de l'Annonciation et de Joliette c'est parce que ces deux institutions décidées au temps de Maurice Duplessis répondent à une question de survie économique de régions défavorisées à fort taux de chômage. Quant au Mont-Providence de Montréal, devenu par la suite le C.H. Rivière-des-Prairies, on le destine 
aux enfants étant donné qu'il est le seul à répondre aux besoins stricts de cette population.

Donc, au lieu de construire de nouveaux asiles, il faut convertir ces lieux d'hébergement « chronicisants » en milieu de traitement humanisant et ouvrir des services de psychiatrie dans les hôpitaux généraux. La psychiatrie commence à avoir pignon sur rue. Les professionnels de la santé mentale se trouvent dans la communauté non pas pour y établir un quadrillage policier-psychiatrique, mais pour traiter le malade mental dans sa famille, dans sa communauté. Ainsi sont mis en place, aussi bien à Montréal, Québec, Sherbrooke, l'Annonciation, Joliette et Trois-Rivières qu'à Sorel, Valleyfield et St-Hyacinthe, des services de remotivation, de réhabilitation sociale et de réentraînement au travail pour des patients chroniques fonctionnels. Des services de thérapie familiale de pré et postcure sont aussi organises. Ainsi, grâce aux médicaments psychotropes et à la psychothérapie devenue possible, un nombre imposant de patients laissent les « donjons » de l'asile pour mener une vie plus "normale " dans un foyer nourricier. Après cette première phase, des professionnels assurent également un service de consultation, voire d'enseignement itinérant auprès des corps professionnels qui s'occupent de près ou de loin de la santé mentale ; ce sont les médecins omnipraticiens, le service social, les commissions scolaires, les unités sanitaires et la Cour du bien-être social.

Cette intervention discrète par personnes ressources interposées était de nature à éviter une psychiatrisation non désirée de toute la vie sociale et à démystifier à long terme la psychiatrie. Mais dans ce vaste mouvement de démocratisation et d'universalisation de l'éducation et des soins de santé que charriait la révolution tranquille, il aurait été mal vu de tenir l'arrière-pays à l'écart des largesses sociétales. Aussi, sous l'égide de la Division des services psychiatriques du Dr Dominique Bédard, on trouva une solution réellement québécoise : les équipes volantes, dont la base de lancement était le C.H. Ste-Justine, le C.H. Rivière-des-Prairies, l'Institut Albert-Prévost de Montréal et des hôpitaux de pointe de la région de Québec ; deux jours par mois ces 
équipes volantes s'envolaient vers Rouyn-Noranda (bassin de population de 60000 habitants), Amos, La Sarre, Val d'Or et Ville-Marie (35 000 habitants), ainsi que vers la Côte Nord et la Gaspésie. Et d'année en année, la disparité régionale psychiatrique reculait, puisque dix ans après, des équipes multidisciplinaires étaient à l'oeuvre en dehors de Montréal et de Québec et que 90 psychiatres y travaillaient.

Ici la psychiatrie communautaire ne s'est pas faite contre l'hôpital psychiatrique. Loin de là. L'hôpital psychiatrique s'est libéré des tâches réclusives auxquelles on l'avait relégué. Sous l'empire de nouvelles idéologies, on procéda à la redéfinition de concepts tels que la maladie mentale, la normalité et la pathologie, l'internement. L'antipsychiatrie en vint même jusqu'à nier le concept de la maladie mentale au point d'en faire un mythe, une pure invention de l'esprit. Mais cet excès est quand même ancré dans la réalité. En effet, jusqu'à récemment, la tâche confiée à l'hôpital psychiatrique était de contenir la folie à l'intérieur des murs de l'hôpital pour protéger la société. On avait pris l'habitude d'élever les édifices de la folie au grand air, loin de la communauté, ce qui devait confirmer à la longue la marginalité autant chez les malades que chez les soignants. L'asile devait défendre les normes « rationnelles » de la société contre l'irrationalité des individus réputés « fous ». À l'autre pôle, il s'est trouvé un courant pour prôner que le fou était en réaction saine contre une situation insupportable et qu'il se libérait de la société par des « transcendances imaginaires ».

En fin de compte nous aboutissons à une alternative à deux termes exclusifs qui suppose une division sectorielle de tout le champ de la santé mentale : le courant psychiatrique, i.e. être avec la société contre le fou, et le courant anti-psychiatrique, i.e. être avec le fou contre la société. Mais cet antagonisme a été dépassé avec le temps. Si on dit couramment que la société façonne les individus, on doit admettre honnêtement que les individus, suivant leur appartenance de classe, bénéficient de pouvoirs inégaux pour façonner en retour cette société à leur guise. Maintenant, et cela de plus en plus, l'hôpital psychiatrique devient un élément dans le réseau de la santé mentale au lieu 
d'être en marge de la société, et on évite par le fait même d'isoler le patient dans l'hôpital et de l'exclure de la vie sociale.

La protection du malade mental est à mettre également au compte des retombées bénéfiques de la révolution tranquille. La loi sur la protection du malade mental promulguée en 1971 a été amendée depuis. La Commission des droits de la personne a demandé qu'elle respecte la charte des droits et libertés de la personne. Ainsi, en 1978, cette commission a recommandé la prudence dans l'utilisation par le clinicien du critère de dangerosité. Elle veut particulièrement modifier l'article 11 de la loi :

Il nous semble évident qu'une personne peut souffrir de désordres mentaux graves qui soient susceptibles de mettre en danger ou la sécurité de cette personne ou d'autrui et à la fois être en mesure d'avoir la capacité de refuser d'une façon éclairée la chimiothérapie ou les électro-chocs.

À l'intérieur même des hôpitaux psychiatriques, le comité des bénéficiaires, espèce d'ombudsman, cherche par tous les moyens, à travers le maquis des procédures de l'appareil médical et du professionnalisme triomphant, à faire respecter les droits fondamentaux du malade mental. Partout, peut-être avec réticence, on tend à reconnaître à ce citoyen la faculté de jouir au même titre que les autres des droits et des privilèges de la société. En tout cas, malgré l'extrême lenteur du gouvernement du Parti Québécois dans ce dossier, le psychiatrisé est en voie de cesser d'être un citoyen diminué, délaissé.

Mais depuis 1971, date de la mise en application de la grande réforme de la santé et des services sociaux par Claude Castonguay, la psychiatrie a perdu de son souffle. Elle avait encore besoin d'un statut particulier durant au moins une décennie pour continuer sa croisière. Mais malgré le mérite et les résultats de la Division des services psychiatriques, le bulldozer de la logique de la production industrielle a tout rasé et la psychiatrie s'est trouvée fondue dans un melting-pot. Il fallait « normaliser » même si la psychiatrie s'occupe de «l'anormal », 
ce qui n'entre pas dans la préoccupation du puissant lobby médicochirurgical, encore moins dans celle des technocrates du ministère des Affaires sociales. Deux ans après, Claude Forget remplace Claude Castonguay à la tête du MAS. Mais la psychiatrie ne connaît guère un meilleur sort. Ce n'est pas à cause de la mauvaise humeur passagère ou des préjugés d'un ministre contre les professionnels de « la médecine des insensés ", mais plutôt à cause d'une logique, celle de contrôler tout le champ social.

À l'exception des corporation professionnelles, comme celle des médecins, qui a réussi au cours de l'histoire à consolider ses positions, l'État québécois a radicalement mis au pas toutes les jeunes corporations qu'il avait contribué à mettre au monde depuis la révolution tranquille, par exemple celle qui regroupe les professions dites « des sciences humaines », celle des administrateurs, etc. Québec en avait marre de toutes ces corporations qui menaçaient, à cause de leur pouvoir, l'application de la réforme Castonguay-Nepveu. Le Québec venait de vivre la grève de la Fédération des médecins spécialistes (1970) et avait encore à l'esprit ce déploiement de la force médicale. l'État québécois avait des comptes à régler avec ces médecins qui, dans l'inconscient collectif, ont le droit de vie et de mort, ces médecins qui ont même accru leur pouvoir lors de la réforme de la santé et des services sociaux en développant une ligne de pouvoir parallèle à l'administration technocratique dans les organismes de santé, les conseils de surveillance professionnelle. Québec ne voyait pas d'un bon oeil ces médecins qui refusaient de déléguer des actes à d'autres professionnels, qui manifestaient leur résistance au salariat, qui boycottaient systématiquement les C.L.S.C. par l'ouverture d'environ 400 polycliniques privées (plus de quatre fois le nombre de C.L.S.C.).

Donc il n'était pas question de laisser se développer au sein du ministère des Affaires sociales des groupes de pression médicale qui, un jour ou l'autre, pourraient devenir puissants. D'où la disparition de la direction des services psychiatriques du Dr Dominique Bédard et par la suite celle du Service aux malades mentaux du Dr Rhéal Lajoie. 
Pour Québec, il fallait non seulement uniformiser, mais aussi contrôler, c'est-à-dire réduire le nombre des détenteurs de pouvoir décisionnel.

Mais il y a quand même des causes internes à cette crise. Le pouvoir s'attaque rarement aux organisations bien portantes. Si Claude Forget a attaqué la sectorisation des soins en psychiatrie comme une entrave aux libertés individuelles, s'il a mis en doute l'efficacité du travail multidisciplinaire, c'est parce que le corps organisationnel des psychiatres était en crise, sinon ses arguments, assez discutables à notre avis, n'auraient eu aucune portée. Il y avait un conflit idéologique au sein de la psychiatrie. L'ordre du jour, c'était de pratiquer la psychiatrie à l'intérieur de l'hôpital général, bref la psychiatrie devait devenir un département comme les autres. Il fallait mettre fin au statut particulier de la psychiatrie et ainsi couper court aux idéologies de gauche qui influençaient surtout les jeunes psychiatres. D'un côté les " anciens », appuyés d'ailleurs par le « lobby » médico-chirurgical, voulaient une psychiatrie plus médicale, plus chimiothérapeutique, bien résolue à ne pas envoyer l'électrochoc au musée de l'horreur. De l'autre côté les «modernes », partisans d'une psychiatrie communautaire multidisciplinaire, défendaient la prévention, les centres de jour, les C.L.S.C. ; c'étaient des psychiatres actifs dans des comités de citoyens qui écrivaient :

La participation des citoyens à la création et à la gestion des outils sociaux dont ils ont besoin pour améliorer leur vie et leur relation interpersonnelle et pour développer leurs talents dans la mesure du possible (...) semble être un des éléments des plus prometteurs en ce qui concerne la santé mentale d'un groupe (Messier, 1973).

En dernier lieu, il faut mentionner une autre réalité qui a pu avoir certains effets sur le moral des troupes. Après le dépôt du rapport Bédard en 1962, Québec avait alloué des crédits importants à la formation des psychiatres dont le pays avait grand besoin. Au point de vue financier, la situation du médecin résident en psychiatrie était des plus enviables. Or, vers 1972, l'État québécois commença à sabrer dans les 
budgets et ces différentes mesures incitatives disparurent, même la prime d'éloignement accordée aux psychiatres œuvrant en milieu rural. Après douze années où le nombre des psychiatres avait crû de $600 \%$, il y eut une certaine baisse des "vocations " psychiatriques chez les finissants de médecine. Dans les années 1965-1968, 10\% des finissants de médecine des universités nord-américaines choisissaient la psychiatrie ; actuellement, le pourcentage est tombé à 3,2\%. Ajoutons que les attaques virulentes des anti-psychiatres ont fait beaucoup de tort à l'image de cette jeune discipline qui avait des problèmes sémiologiques évidents (diagnostic) tout en défendant pouce par pouce son terrain menacé par l'impérialisme psychanalytique.

Et la crise continue. En janvier 1979 sort le rapport du Comité de la psychiatrie du Québec, présidé par le docteur Jacques Mackay. Son titre en dit long: Situation de la psychiatrie au Québec: lacunes et perspectives. Il provoque une réaction négative de la part de la majorité des psychiatres et aussi de certains groupements comme la Corporation professionnelle des travailleurs sociaux du Québec, qui critique le fond du rapport. Ses propositions marquent un certain recul, par exemple l'échange de la multidisciplinarité pour le système hiérarchique traditionnel, la réduction du concept de santé mentale au concept étroit de psychiatrie, et même le type d'organisation définie. D'ailleurs les psychiatres n'ont pas tous apprécié l'arrivée des autres intervenants dans le champ pourtant vaste de « la folie » et craignent les définitions sociologiques de ce phénomène (voir le Rapport Bélanger, 1979).

Les chefs de file de la psychiatrie s'inquiètent et soulèvent le débat dans les centres hospitaliers. On écrit beaucoup (Maurice Dongier, «La psychiatrie des années 80, panorama des problèmes et discussions »; Dominique Bédard, « Réflexions sur l'organisation des services psychiatriques au Québec »), et il suffit de feuilleter le bulletin de l'Association des psychiatres du Québec pour s'en convaincre. D'ailleurs, le thème général du XVe congrès de l'Association des psychiatres du Québec, tenu au Manoir Richelieu, à Pointe-au-Pic, dans le comté de Charlevoix, en juin 1981, témoigne de cette inquiétude: 
«Les vents qui soufflent au Québec en psychiatrie... » Oui, mais il faudrait que ces vents aient un écho jusqu'à l'édifice du 1075, Chemin Ste-Foy, à Québec, qui abrite les technocrates du ministère des Affaires sociales.

\section{Réflexions sur les pratiques actuelles}

Retour à la table des matières

Étant donné que le congrès de l'ACSALF est centré cette année sur une sociologie des lieux de pratique, nous allons tracer quelques axes de réflexion autour desquels s'effectuent nos pratiques. La description en a déjà été faite ailleurs (H. Dorvil, 1979 ; R. Mayer, 1980). Dans les années soixante, l'asile a subi les feux de la critique. Les institutions étaient décrites comme un monde kafkaïen, un univers concentrationnaire qui traitait la folie par l'exclusion, comme un dérangement à l'idéologie de la société industrielle. L'asile a réagi en ouvrant ses portes et surtout en gavant ses pensionnaires de gâteries. Mais une chaîne en or demeure une chaîne. C'est vrai qu'il existe des dizaines de professionnels qui surprotègent les patients, c'est vrai que l'asile a transformé sa chapelle en gymnase, c'est vrai que les zones de détente ne manquent pas : bibliothèque, cinéma, bal du samedi soir, commerce de drogue, d'alcool, de sexe, voire bientôt une piscine de dimensions olympiques au C.H. Louis-Hippolyte-Lafontaine, un « engagement électoral » du ministre Lazure. Alors pourquoi le malade mental accepterait-il de retourner dans la société, cette société qui lui en offre beaucoup moins que l'asile, sans compter les préjugés qu'elle continue à entretenir à son égard ? Le patient a compris. D'ailleurs, une étude de Mme Andrée Melançon-Ouellette vient de nous apprendre que près de $80 \%$ des Québécois sont pour le maintien de l'asile, non pour eux, non pour leur famille, mais pour autrui.

Nous disons donc que l'asile a poussé le patient à ce choix schizophrénique, à ce refus de la société d'où il vient. Certains patients pas- 
sent plusieurs années dans une " unité de soins » sans être l'objet d'aucun projet thérapeutique. Ensuite le patient qui vit à l'hôpital est souvent l'objet d'un rejet familial. Il en découle que l'institution en arrive à représenter tout pour lui. Et c'est là le point de rupture, le point de non-retour, car il faut absolument sauvegarder des relations avec l'extérieur, quelque conflictuelles ou nocives qu'elles puissent être. Il faut un lien autre, un lien différent, alternatif, pour que le patient ait le choix entre deux systèmes. D'où la nécessité pour le patient de laisser l'asile quelques jours par mois pour vivre dans une autre situation. Mais depuis les années soixante-dix, c'est le règne de la gestion : la finalité de l'institution n'est plus de soigner mais d'être gérée.

Les médicaments psychotropes ont été pour beaucoup dans la transformation du visage de l'asile et dans le retour massif des handicapés mentaux dans la communauté. Les chiffres sont assez éloquents : à St-Jean-de-Dieu, par exemple, il y avait en décembre 1960 5600 « malades », alors qu'en décembre 1980, il n'en reste plus que 2 200. L'État québécois a trouvé là un moyen pour réduire le coût astronomique du fonctionnement des hôpitaux des années soixante-dix, le prix de journée des familles d'accueil étant 10 fois inférieur à celui des institutions hospitalières.

La plupart des patients sont retournés dans la communauté, assez souvent sans préparation ; certains vivent dans des familles d'accueil, genre de mini-succursales des C.H., alors que d'autres traînent dans les gares ou vagabondent sur les grands chemins.

En milieu rural, ce n'est pas la place qui manque. Assez souvent ces patients remplacent, ni plus ni moins, les nombreux enfants partis en ville gagner leur vie. Pensez à toutes ces grandes maisons canadiennes-françaises qui sont aujourd'hui remplies et servent une nouvelle fois la communauté. Sans nier l'incitatif financier, ce qui mérite ici d'être souligné, c'est l'attachement de toutes ces personnes à leur patient, qui devient un membre à part entière de la famille, et aussi le peu de difficultés rencontrées lorsqu'il s'agit de convaincre les leaders 
de la communauté, les associations bénévoles, toute la population de faire une niche à l'handicapé mental.

La dernière réflexion concerne l'inégalité des êtres humains devant la maladie et la mort. Comme une fatalité, il existe encore une surreprésentation des membres de la classe défavorisée chez les malades mentaux. Les coussins amortisseurs des tensions axiogènes sont inégalement répartis dans nos sociétés. Les cadres d'entreprise, les professionnels stressés vont se reposer dans les Laurentides par exemple ou prennent l'avion en direction du sud, alors que l'ouvrier en butte au stress va « toffer » son ouvrage jusqu'à ce qu'il pète.

Si la maladie est déclarée, le trajet sera aussi différent. Le bourgeois canadien-français ira se remettre de sa dépression à l'Institut Albert-Prévost, le canadien-anglais au Allen Memorial Institute, ou les deux iront cacher leurs faiblesses psychiques dans les cliniques privées de Floride ou de Californie. Il faut protéger l'image de Narcisse, sa crédibilité... Quant aux assistés sociaux, ils prendront le chemin de l'asile, le C.H. Louis-Hippolyte-Lafontaine pour le francophone, le C.H. Douglas de Verdun pour l'anglophone, et ce à la risée du quartier et des copains de travail. Cette relation « classes sociales/maladies mentales " s'avère d'autant plus importante que nous vivons une époque de médicalisation de la vie, de traitement individualiste et médical des problèmes collectifs de santé, où l'on évacue cavalièrement l'influence pathogène des conditions de vie parce que ça renvoie à des luttes politiques.

Actuellement, c'est le mouvement alternatif qui porte le flambeau de la contestation sociologique de cette visée individualiste et culpabilisante. En effet, nous voyons un lien direct entre la croissance des formes de désorganisation mentale, le taux record d'admissions, de réadmissions surtout de jeunes d'à peine vingt ans et les conditions de vie et de travail aberrantes, le chômage grandissant, une compétition de plus en plus effrénée, l'écart entre les désirs chauffés à blanc par la publicité de l'abondance et la probabilité réelle pour les petites gens de 
les satisfaire. Bien souvent ces personnes ne comprennent pas le mécanisme de fonctionnement des rapports sociaux et se croient tout de go responsables de leur chômage, de leur pauvreté, de leur rejet, de leurs tensions familiales, de leurs échecs. « La folie survient au moment où un individu ayant intériorisé toutes ces invalidations, développe une pensée et un mode d'être, basés principalement sur ces invalidations ; ce qui l'entraîne à fuir la réalité où il ne peut satisfaire ses besoins, et il utilise les outils de fuite que son milieu lui propose : médicaments, alcool, drogue, spiritualisme, etc. » (Maison St-Jacques, 1981).

Bref, la recherche de "pratiques alternatives » est tout aussi présente, sinon plus, dans le champ des pratiques psychiatriques que dans les autres champs d'intervention sociale au Québec. Des organismes, tels que le groupe Solidarité-psychiatrie (1980) ou encore celui du Projet d'aide logement (P.A.L.) de l'hôpital Douglas (1980), etc., font appel particulièrement à la participation des patients et se situent plutôt dans la tradition des groupes d'entraide. Ces groupes, à vrai dire, s'intéressent peu à l'élaboration d'un discours politique ; ils sont plutôt intéressés à développer des structures d'accueil et de support afin de faciliter l'intégration d'ex-patients psychiatriques. D'autres intervenants tentent de mettre au point un nouveau type de prise en charge qui implique le patient, son réseau et l'équipe d'intervention à travers une approche bio-psycho-sociale (Desmarais et al., 1980 ; Desmarais et Mayer, 1980 ; Mayer et Desmarais, 1980 ; Daher, 1980). La notion de réseau se réfère ici au réseau primaire du patient identifié, i.e. à la totalité des parents, amis, compagnons de travail, voisins, etc., qui communiquent entre eux sur une base d'affinités personnelles, et ce, afin de susciter une prise en charge plus collective du problème de la maladie mentale.

La recherche de " pratiques alternatives » est particulièrement forte parmi ceux qui interviennent plus spécifiquement auprès des femmes et insistent sur l'incidence de la maladie mentale chez la femme et la nécessité de thérapies proprement féministes. 
Finalement, mentionnons un certain nombre d'initiatives qui datent de l'époque des années soixante-dix et de la vogue des comités de citoyens : Clinique des citoyens de St-Jacques (1980) ; Clinique populaire de Pointe-St-Charles (Blanchet, 1978) et plus récemment l'initiative de la Maison St-Jacques (1981). Ces organisations se caractérisent par le fait qu'elles tentent de susciter une participation des citoyens et qu'elles abordent la maladie mentale à partir d'une problématique socio-politique de la santé... De façon générale elles dénoncent la «mentalité asilaire » qui prévaut encore trop souvent dans les institutions psychiatriques québécoises et qui maintient une distance artificielle entre soignants et soignés. Parmi les principaux objectifs poursuivis par ces organismes on peut mentionner l'appréhension de la maladie mentale à partir de trois niveaux complémentaires (soit le biologique, le psychologique et le socio-politique) ; l'utilisation optimale du potentiel thérapeutique de chaque individu; la déspécialisation des problèmes rencontrés ; la démystification des statuts sociaux et la déprofessionnalisation des rapports soignants-soignés (Blanchet, 1978, p. 4). Conséquemment, on insiste beaucoup sur l'implication réelle et concrète des soignants avec les soignés dans un processus de changement social. Finalement, les problèmes de santé physique ou mentale sont souvent mis en relation avec les caractéristiques du mode de production en général et avec les conditions de vie ou de travail en particulier.

En théorie et en pratique, cette nouvelle approche permet à la Maison St-Jacques, par exemple, d'offrir à 125 jeunes (de 18-30 ans) la possibilité de se réapproprier une identité et de se lier socialement. Par leur implantation dans les quartiers populaires, leur implication dans les organismes communautaires, leur disponibilité à dialoguer avec tous et leur franche collaboration avec les autres intervenants du milieu institutionnel, les trente unités du mouvement alternatif à la psychiatrie ne constituent plus une marginalité mais un définissement majeur dans le champ de la santé mentale. Ce n'est pas pour rien qu'il existe au MAS un comité du mouvement alternatif à l'ombre de la tou- 
te nouvelle direction du service des programmes de santé mentale. Les grilles d'analyse devront donc se fignoler à nouveau pour tenir compte de cette nouvelle réalité... non monolithique.

\author{
Robert Mayer \\ Université de Montréal \\ École de service social
}

Henri Dorvil

C.H. Louis-Hippolyte-Lafontaine (C.S.S.M.M.)

\title{
Bibliographie
}

$\underline{\text { Retour à la table des matières }}$

G. Aird et A. Amyot, « La psychiatrie communautaire », in Lalonde et Grunberg (1980).

L. Blanchet, « La santé mentale à Pointe St-Charles : vers une prise en charge collective », Santé mentale au Québec, vol. 3, no 1, 1978.

Françoise Boudreau, "The Quebec psychiatric system in transition », La Revue canadienne de sociologie et d'anthropologie, vol. 17, no 1, février 1980, p. 122-138.

J.F. Chapdelaine, « Le rôle du travailleur social dans l'équipe multidisciplinaire en milieu de santé », Intervention, no 48, p. 7-12.

Clinique des citoyens de St-Jacques, «Comment défendre la santé du peuple et sa santé mentale », Santé mentale au Québec, vol. 5, no 1, juin 1980, p. 47-54. 
Collectif Les Équipes Volantes, La Psychiatrie communautaire dans le NordOuest Québécois, Collection «Équipes psychiatriques pour adultes et enfants », mars 1969 -décembre 1970, p. 10.

P. Daher, « Les pratiques de réseaux : recherche-action », Intervention, no 58, 1980, p. 13-24.

D. Desmarais et R. Mayer, « Le modèle d'intervention auprès du réseau (illustration et commentaire) ", Santé mentale au Québec, vol. 5, no 1, juin 1980, p. 22-41.

D. Desmarais, C. Hamel, R. Dauphinais, L. Roy et C. Sterlin, « Un modèle bio-psycho-social d'intervention en réseau », Service social, 29, 3, 1980.

H. Dorvil, « Intervention socio-psychiatrique à Berceauville », Revue internationale d'action communautaire, printemps 1979, p. 43-51.

Alain Fugère, « La stratégie matérialiste d'Henry Howard, médecin aliéniste québécois (1815-1887) », Santé mentale au Québec, vol. 3, no 2, nov. 1978, p. 27-45.

Pierre Harvey, « Préliminaires à une sociologie historique des maladies mentales au Québec », Recherches sociographiques, vol. 16, no 1, 1975.

M.L. Lachapelle, «Faut-il sonner le glas... de la psychiatrie communautaire ? » Les Cahiers du psychologue québécois, vol. 2, no 1, 1979, p. 20-23.

Pierre Lalonde et Frédéric Grunberg (sous la direction de), Psychiatrie clinique. Approche contemporaine, Chicoutimi, G. Morin Éditeur, 1980, p. 872. 
R. Mayer et D. Desmarais, «Réflexion sur la recherche-action : l'expérience de l'équipe d'intervention en réseau de l'hôpital Douglas à Montréal », Service social, 29 : 3, 1980.

Maison St-Jacques, Textes de présentation, Montréal 1981, miméo, $30 \mathrm{p}$.

Andrée Melançon-Ouellette, Étude sur les connaissances et les perceptions des services psychiatriques au Québec, Québec, Ministère des Affaires sociales, 1980, 185 p.

Michel Messier, Le Rôle du CL.S.C. dans l'organisation des soins psychiatriques, texte présenté au congrès de l'Association des psychiatres du Québec, 1973.

J.-C. Pagé, Les Fous crient au secours, Montréal, Éd. du Jour, 1961, 117 p.

André Paradis et collaborateurs ; "L'émergence de l'asile québécois au 19e siècle ", Santé mentale au Québec, vol. 2, no 2, nov. 1976, p. 2-44.

André Paradis, «L'asile temporaire de Toronto (1841-1850) ou l'impossibilité provisoire de l'utopie asilaire », Santé mentale au Québec, vol. 3, no 1, juin 1978, p. 18-36.

Marc Renaud et ai., Médecine et société : les années 80, Montréal, Éd. coopératives Albert St-Martin, 1981.

Rodrigue Samuel, Aperçu historique des asiles d'aliénés dans la Province de Québec entre 1845 et 1890, Université Laval, Institut supérieur des sciences humaines, mai 1974, 25 p., texte miméo.

Yanick Villedieu, «Les nouveaux asiles », Québec Science, sept. 1979, p. 16-21. 
Alain Vinet, «La vie quotidienne dans un asile québécois », $R e$ cherches sociographiques, vol. 16, no 1, 1975, p. 85-112.

Hubert Wallot, "Perspective sur l'histoire québécoise de la psychiatrie : le cas de l'asile de Québec », Santé mentale au Québec, vol. 4, no 1, juin 1979, p. 102-123.

Le Comité de la psychiatrie du Québec, Situation de la psychiatrie au Québec : lacunes et perspectives d'organisation, Montréal, 22 février 1979, $42 \mathrm{p}$.

C.P.T.S.Q., Commentaires et recommandations sur le rapport. Le Comité de la psychiatrie du Québec, 11 juin 1980, 10 p.

Fin du texte 